\title{
Mentoria entre pares: percepções de suporte social e ambiente educacional de estudantes de medicina
}

\section{Peer mentoring: medical students' perceptions of social support and the educational environment}

\author{
Natália Dilella Acherman' (1) | nataliaacherman@gmail.com \\ Adalgisa Peixoto Ribeiro' 1 adalpeixoto@yahoo.com.br \\ Lucas Martins de Lima' @ lucasmlima@ufmg.br \\ Ana Carolina Damasceno Cavalcanti ${ }^{2}$ (1) anacarolinadc@gmail.com \\ Thassiane Kelly Quintão Miranda' (1) thassiane_miranda@yahoo.com.br \\ Graziella Lage Oliveira' (1) grazilage.oliveira@gmail.com
}

\begin{abstract}
RESUMO
Introdução: Programas de mentoria entre pares são apontados como suporte efetivo na transição dos estudantes para o ensino superior, colaborando com sua saúde mental e seu desempenho acadêmico.

Objetivos: Este estudo teve como objetivos mensurar e comparar a percepção de suporte social e avaliação do ambiente educacional entre estudantes membros e não membros do Grupo de Estudos em Didática Aplicada ao Aprendizado da Medicina (Gedaam), no curso de graduação em Medicina da Universidade Federal de Minas Gerais (UFMG).

Método: Trata-se de estudo transversal que utilizou dados do cruzamento entre os bancos da pesquisa QualiMed e dos registros do Gedaam. Dos 1.470 estudantes que participaram do inquérito QualiMed, 347 eram vinculados ao Gedaam. A percepção do suporte social foi mensurada pela Escala de Satisfação com o Suporte Social (ESSS); e a avaliação do ambiente educacional, pelo Dundee Ready Education Environment Measure (DREEM). Realizaram-se análises descritivas e comparativas univariadas por meio do qui-quadrado de Pearson e do teste $t$ de Student, considerando um valor-p $<$ 0,05 para verificação da significância estatística. Os dados foram analisados no software Statistical Package for Social Sciences (SPSS), versão 19.0.

Resultado: Verificou-se diferença estatisticamente significativa entre membros e não membros nas variáveis ciclo do curso, sexo, orientação sexual, recebimento de bolsas de auxílio e prática de atividade física regular. Os participantes do Gedaam apresentaram pior percepção quando comparados a não membros em todos os domínios da ESSS, exceto para a satisfação com atividades sociais (valor-p < 0,05). 0 ambiente educacional foi avaliado pelos dois grupos como tendo "muitos problemas" (escore = 95,51 para membros Gedaam e 100,05 para não membros), com diferença estatisticamente significativa.
\end{abstract}

Conclusão: Observou-se um perfil mais crítico em relação à percepção de suporte social e ao ambiente acadêmico entre os estudantes. Sugere-se que a mentoria entre pares auxilie positivamente o percurso acadêmico e pessoal dos estudantes de Medicina.

Palavras-chave: Grupo de Pares; Suporte Social; Educação de Graduação em Medicina; Estudantes de Medicina.

\section{ABSTRACT}

Introduction: Peer mentoring programs are seen as effective support for student transition to higher education, contributing to their mental health and academic performance.

Objective: To measure and compare the perception of social support and evaluation of the educational environment among student members and non-members of the Grupo de Estudos em Didática Aplicada ao Aprendizado da Medicina (Study Group in Didactics Applied to Learning in Medicine GEDAAM), on the undergraduate medicine course at the Federal University of Minas Gerais (UFMG).

Method: Cross-sectional study using data from the crossover between QualiMed research data and GEDAAM records. Of the 1,470 students who participated in the QualiMed survey, 347 were associated to GEDAAM. The perception of social support was measured by the Social Support Satisfaction Scale (ESSS) and the educational environment assessment by the Dundee Ready Education Environment Measure (DREEM). Univariate descriptive and comparative analysis were performed using Pearson's Chi-square and Student's t-test, considering a p-value $<0.05$ to verify statistical significance. Data were analyzed using Statistical Package for Social Sciences (SPSS) software, version 19.0.

Results: A statistically significant difference was found between members and non-members in course stage, sex, sexual orientation, receipt of aid grants and practice of regular physical activity. GEDAAM participants had a worse perception when compared to non-members in all ESSS Domains, except for satisfaction with social activities ( $p$-value $<0.05$ ). The educational environment was assessed by both groups as having "many problems" (score $=95.51$ for GEDAAM members and 100.05 for non-members), with a statistically significant difference.

Conclusion: A more critical profile was observed in relation to the perception of social support and the academic environment among students. It is suggested that peer mentoring positively helps the academic and personal path of medical students.

Keywords: Peer Group; Social Support; Medical Education Undergraduate; Medical Students.

${ }^{1}$ Universidade Federal de Minas Gerais, Belo Horizonte, Minas Gerais, Brasil.

2 Universidade Federal dos Vales do Jequitinhonha e Mucuri, Teófilo Otoni, Minas Gerais, Brasil.

Editora: Lia Marcia Cruz da Silveira

Recebido em 27/02/21; Aceito em 03/03/21. Avaliado pelo processo de double blind review. 


\section{INTRODUÇÃO}

O ingresso no ensino superior é marcado por diversas mudanças biopsicossociais que podem influenciar na qualidade de vida e no desenvolvimento de transtornos mentais. Vários estudos demonstram maior incidência de depressão, ansiedade e outros transtornos psiquiátricos em estudantes de Medicina, quando comparados a alunos de outros cursos $^{1,2}$. No Brasil, a prevalência de depressão e ansiedade nesses estudantes é alta $(30,6 \% \text { e 32,9\%, respectivamente) })^{3}$. Tal cenário está atrelado a diferentes fatores estressores aos quais os acadêmicos de Medicina estão expostos: carga horária extensa, amplo volume de informações a serem aprendidas, ambiente de competitividade entre os colegas, dificuldades de gestão do tempo e de harmonização da vida acadêmica com vida pessoal, além do medo de cometer erros ${ }^{4}$.

Diante desse contexto, a adoção de estratégias que visem auxiliar a inserção do estudante no contexto universitário faz-se necessária. Uma das ferramentas que vêm ganhando espaço nas escolas médicas é a mentoria, em que um indivíduo com mais experiência em determinada área orienta uma outra pessoa, menos experiente, com o objetivo de ajudá-la a atingir melhor desenvolvimento pessoal e/ou profissional. A mentoria pode ocorrer de forma clássica entre professores e estudantes, e também entre pares (peer mentoring). O conceito de mentoria ou mentoring tem evoluído, e, no contexto atual, ela pode ser traduzida como uma parceria de aprendizagem recíproca por meio do estabelecimento de interações empáticas e de confiança ${ }^{5-7}$.

Nas literaturas nacional e internacional, são encontradas evidências do valor da mentoria entre pares na adaptação do acadêmico ao ensino superior, no desenvolvimento pessoal e profissional, na diminuição do estresse e na facilitação no processo de transição ao ensino superior ${ }^{8}$. A maior facilidade de os estudantes expressarem seus sentimentos a seus pares pode contribuir para o estabelecimento de uma relação mais amigável, favorecendo maior compreensão mútua e a abordagem de temas como saúde mental e bem-estar ${ }^{9}$. Alguns estudos destacam que a mentoria entre pares ajuda a desvendar o "currículo oculto", a negociar acesso aos recursos e a identificar aspectos não cobertos formalmente pelo currículo do curso ${ }^{10,11}$.

Além disso, os programas de mentoria desempenham um papel importante na formação médica, pelo fato de valorizar todos os saberes requeridos pela profissão médica componentes afetivos, éticos, sociais e cognitivos ${ }^{12}$. A mentoria também é responsável por proporcionar um ambiente de ajuda mútua e suporte social, entendido como a percepção subjetiva de cada indivíduo sobre a disponibilidade de pessoas em relações sociais de apoio ${ }^{13}$, ou seja, tanto o mentor quanto o mentorando se beneficiam nesse processo.
Considerando a importância da mentoria entre pares para a formação médica, o presente estudo teve como objetivos mensurar e comparar a percepção de suporte social e avaliação do ambiente educacional, entre estudantes membros e não membros do Grupo de Estudos em Didática Aplicada ao Aprendizado da Medicina (Gedaam), no curso de graduação em Medicina da Universidade Federal de Minas Gerais (UFMG).

\section{MÉTODO}

\section{Sobre o Gedaam}

O Gedaam é um grupo de mentoria entre pares criado em 2013, na Faculdade de Medicina da UFMG. Iniciou suas atividades com o objetivo de propiciar um ambiente "seguro", dentro da universidade, para se aprender a aprender. Nesse ambiente, caberiam o erro, o compartilhamento de técnicas e experiências e a promoção de um percurso acadêmico de excelência, contribuindo para o bem-estar do estudante.

Sustenta-se em três pilares:

- Técnica e gestão: nesse pilar, discute-se a educação baseada em evidências, de acordo com os estudos da neurociência e da psicologia do aprendizado, as técnicas de comunicação e apresentação, a gestão do tempo, os recursos educacionais e os desafios ao longo do percurso acadêmico.

- Casos clínicos: foca o treinamento do raciocínio clínico e a contextualização do conteúdo teórico desde o ciclo básico do curso.

- Saúde física e mental: baseia-se em discussões temáticas e no ambiente coletivo de suporte social mútuo.

A estrutura organizacional do Gedaam é composta por coordenadores e participantes. Os coordenadores são estudantes de períodos mais avançados que passaram pelo curso de Formação de Coordenadores, no qual são repassados ensinamentos que compõem o núcleo de aprendizados coletivos em relação às temáticas do grupo. Os participantes são estudantes de diversos períodos do curso de Medicina (em sua maioria) e das demais áreas de ensino superior. A entrada de novos integrantes é feita semestralmente, de forma gratuita, por meio da participação em um evento de abertura (geralmente no formato de simpósio ou aula magna). Ingressam semestralmente em torno de 150 participantes, divididos em subgrupos compostos por oito a 14 membros. As mentorias ocorrem semanalmente com encontros entre uma e duas horas, sob coordenação de até três estudantes mentores. Em todos os subgrupos, são desenvolvidos os temas básicos dos pilares supracitados, com cronograma personalizado e flexível conforme as peculiaridades dos membros. Cabe ressaltar ainda que o Gedaam vem se expandindo para outras instituições de ensino superior (IES). 


\section{Desenho do estudo}

Os dados do presente artigo são provenientes do cruzamento de dois bancos de dados: 1. estudo transversal QualiMed, realizado em 2018, com 1470 estudantes de Medicina da UFMG (74,2\% do total de matriculados), de todos os períodos do curso, e 2. registros da estratégia de mentoria entre pares do Gedaam.

O cruzamento entre os bancos foi efetuado por meio de uma variável comum aos dois bancos de dados: o número de matrícula. Assim, foi composto um único banco. Do total de estudantes que participaram da primeira fase do inquérito QualiMed, 347 eram vinculados ao Gedaam, como participantes atuais ou ex-participantes (Figura 1).

\section{Coleta e análise dos dados}

Os dados foram coletados eletronicamente, por meio do Google Forms, entre junho e dezembro de 2018. O questionário da pesquisa continha informações sociodemográficas, relacionadas ao curso, comportamentos, uso de substâncias, saúde mental e percepção sobre a qualidade de vida, o ambiente educacional e o suporte social.

A percepção sobre o suporte social foi avaliada por meio da Escala de Satisfação com o Suporte Social $\left(\right.$ ESSS) ${ }^{14}$, adaptada para o Brasil por Marôco et al. ${ }^{15}$. Esse instrumento possui 15 itens avaliados por escala do tipo Likert de cinco pontos, sendo o menor valor correspondente a "discordo totalmente" e o maior valor a "concordo totalmente". A pontuação total varia de 15 a 75 pontos. Não há ponto de corte para considerar o suporte social deficitário, entretanto, quanto maior o valor total, melhor é a percepção do suporte social. Os autores propõem quatro domínios para melhor análise da escala: 1 . satisfação com amizade, 2. intimidade, 3. satisfação com família e 4. atividades sociais.

A percepção do ambiente educacional foi avaliada por meio do Dundee Ready Education Environment Measure (DREEM), um instrumento projetado especificamente para estudantes de Medicina e outros profissionais de saúde, validado no Brasil por Oliveira Filho ${ }^{16}$. O DREEM possui 50 questões fechadas, distribuídas em cinco domínios, que avaliam a percepção dos alunos sobre: aprendizado, professores, ambiente acadêmico, atmosfera e social. Cada um dos itens recebe uma pontuação Likert que varia de zero (discordo totalmente) a 4 (concordo totalmente). Também é possível obter uma avaliação única considerando todos os 50 itens da escala (escala global), cujos escores variam de zero a 200 pontos (quanto maior o escore, mais positiva a avaliação do ambiente educacional) e podem ser interpretados como: 0-50 = ambiente educacional muito

Figura 1. Fluxograma das etapas do estudo QualiMed e da seleção da amostra da interface entre QualiMed e banco de dados da mentoria entre pares Gedaam.

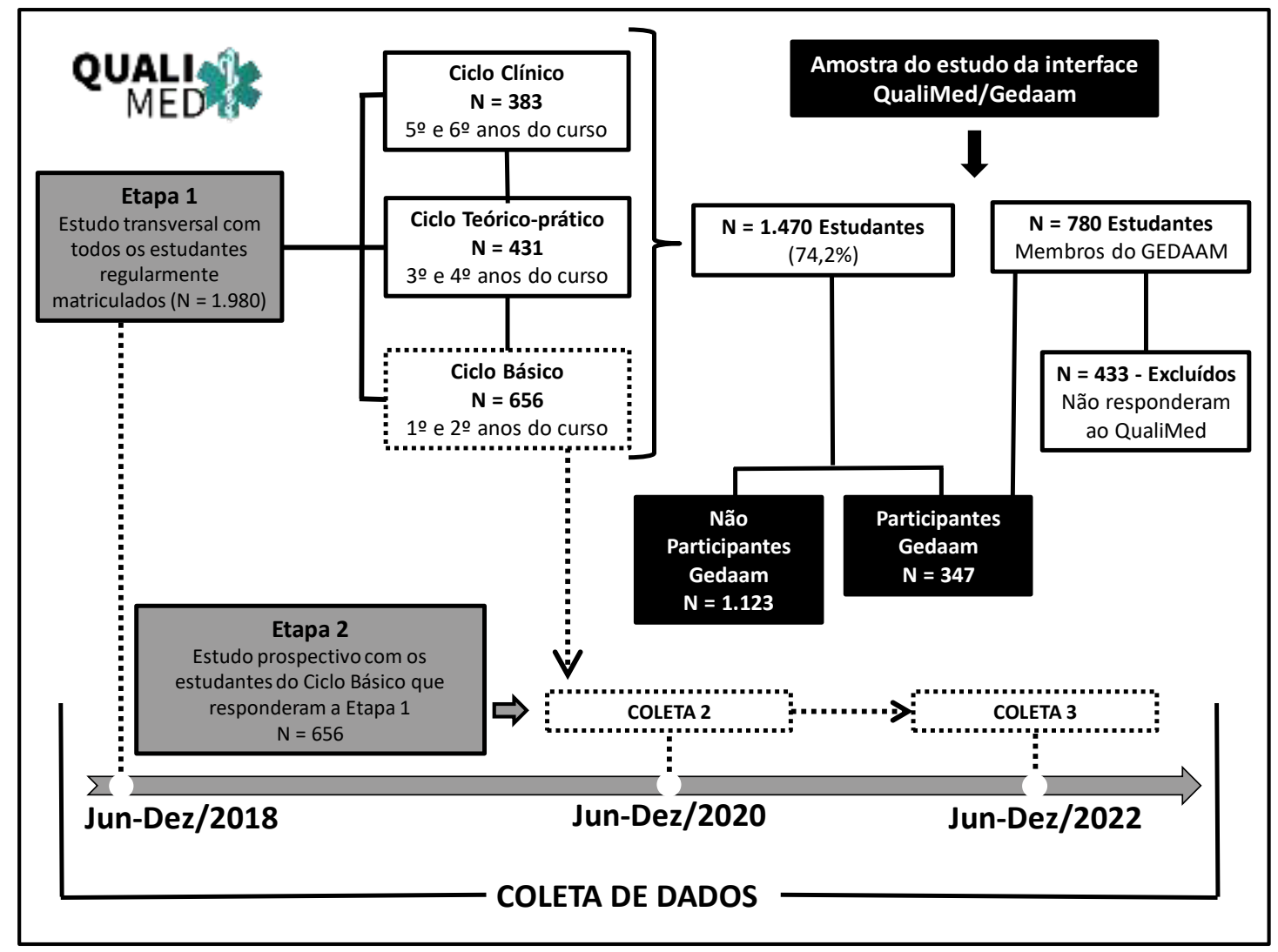

Fonte: Adaptada do projeto de pesquisa QualiMed. 
pobre; $51-100=$ com muitos problemas, $101-150=$ mais positivo do que negativo, $151-200=$ excelente $^{17}$. Os valores mínimo e máximo dos escores para cada domínio diferem de acordo com o número de itens, mas, no geral, a classificação segue categorização semelhante à da escala global.

Foram realizadas análises descritivas e comparativas relacionadas ao perfil dos estudantes, à percepção de suporte social (para itens e domínios) e à percepção do ambiente acadêmico, de acordo com a participação no Gedaam. Para as análises comparativas univariadas, utilizaram-se o qui-quadrado de Pearson para as variáveis categóricas e o teste $t$ de Student ou Mann-Whitney para as variáveis contínuas, dependendo da distribuição encontrada. Todas as análises foram realizadas no programa Statistical Package for Social Science (SPSS), versão 19.0.

O estudo respeitou todos os preceitos éticos, e todos os participantes concordaram com o Termo de Consentimento Livre e Esclarecido (TCLE) de forma on-line. O estudo foi aprovado pelo Comitê de Ética em Pesquisa da UFMG Certificado de Apresentação para Apreciação Ética (CAEE) no 85690218.8.0000.5149 e Parecer n².608.649.

Tabela 1. Perfil dos estudantes de Medicina membros e não membros do Gedaam ${ }^{1}$ que responderam ao QualiMed, Universidade Federal de Minas Gerais, 2018

\begin{tabular}{|c|c|c|c|c|c|c|}
\hline \multirow{3}{*}{ Características } & & \multicolumn{5}{|c|}{ Membros do Gedaam² } \\
\hline & & \multicolumn{2}{|c|}{$\operatorname{Sim}(n=347)$} & \multicolumn{2}{|c|}{ Não (n = 1.123) } & \multirow[b]{2}{*}{$p^{3}$} \\
\hline & & $\mathbf{N}$ & $\%$ & $\mathrm{~N}$ & $\%$ & \\
\hline \multirow{3}{*}{ Ciclo do curso ${ }^{4}$} & Básico & 224 & 64,7 & 432 & 39,8 & \multirow{3}{*}{$<0,001$} \\
\hline & Teórico-prático & 101 & 29,2 & 330 & 30,4 & \\
\hline & Clínico & 21 & 6,1 & 324 & 29,8 & \\
\hline \multirow{2}{*}{ Situação no curso } & Regular & 319 & 91,9 & 1055 & 93,9 & \multirow{2}{*}{0,116} \\
\hline & Irregular & 28 & 8,1 & 68 & 6,1 & \\
\hline \multirow{2}{*}{ Cor da pele } & Branca & 209 & 60,2 & 705 & 62,8 & \multirow{2}{*}{0,214} \\
\hline & Não branca ${ }^{5}$ & 138 & 39,8 & 418 & 37,2 & \\
\hline \multirow{2}{*}{ Sexo } & Feminino & 202 & 58,2 & 541 & 48,3 & \multirow{2}{*}{0,001} \\
\hline & Masculino & 145 & 41,8 & 578 & 51,7 & \\
\hline \multirow{2}{*}{ Orientação sexual ${ }^{6}$} & Heterossexual & 259 & 74,9 & 913 & 81,5 & \multirow{2}{*}{0,005} \\
\hline & Não heterossexual & 87 & 25,1 & 207 & 18,5 & \\
\hline \multirow{2}{*}{ Pratica alguma religião? } & Sim & 167 & 48,4 & 551 & 49,2 & \multirow{2}{*}{0,417} \\
\hline & Não & 178 & 51,6 & 568 & 50,8 & \\
\hline \multirow{2}{*}{ Recebe bolsa de auxílio da universidade ${ }^{7}$} & Sim & 99 & 28,7 & 222 & 19,9 & \multirow{2}{*}{$<0,001$} \\
\hline & Não & 246 & 71,3 & 892 & 80,1 & \\
\hline \multirow{2}{*}{ Curso de Medicina era sua primeira opção? } & Sim & 254 & 73,2 & 854 & 76,0 & \multirow{2}{*}{0,157} \\
\hline & Não & 93 & 26,8 & 269 & 24,0 & \\
\hline \multirow{2}{*}{ Pratica atividade física regular ${ }^{8}$} & Sim & 178 & 51,3 & 635 & 56,5 & \multirow{2}{*}{0,049} \\
\hline & Não & 169 & 48,7 & 488 & 43,5 & \\
\hline \multirow{2}{*}{ Qualidade de vida autorreferida } & Pior percepção & 280 & 80,7 & 883 & 78,6 & \multirow{2}{*}{0,227} \\
\hline & Melhor percepção & 67 & 19,3 & 240 & 21,4 & \\
\hline \multirow{2}{*}{ Sintomas de depressão ${ }^{9}$} & Sim & 142 & 40,9 & 409 & 36,4 & \multirow{2}{*}{0,074} \\
\hline & Não & 205 & 59,1 & 714 & 63,6 & \\
\hline \multirow{2}{*}{ Sintomas de ansiedade elevada ${ }^{10}$} & Sim & 65 & 18,7 & 170 & 15,2 & \multirow{2}{*}{0,069} \\
\hline & Não & 282 & 81,3 & 950 & 84,8 & \\
\hline
\end{tabular}

${ }^{1}$ Gedaam: Grupo de Estudos em Didática Aplicada ao Aprendizado da Medicina.

${ }^{2}$ Estudantes que eram ou haviam sido membros do Gedaam entre os estudantes que responderam ao QualiMed.

${ }^{3}$ Valor-p obtido por meio do teste qui-quadrado de Pearson.

${ }^{4}$ Ciclo básico: corresponde aos dois primeiros anos do curso; ciclo teórico-prático: terceiro e quarto anos; ciclo clínico: quinto e sexto anos.

${ }^{5}$ Cor da pele não branca incluiu autodeclarados negros, pardos, indígenas e outros.

${ }^{6}$ Orientação sexual não heterossexual incluiu homossexuais, bissexuais e assexuais.

${ }^{7}$ Bolsas de apoio logístico e financeiro a estudantes fornecidas pela Fundação Universitária Mendes Pimentel (Fump).

${ }^{8}$ Atividade física regular: aquela praticada por pelo menos 20 minutos, no mínimo de duas a três vezes por semana.

${ }^{9}$ Obtido por meio da Escala de Sintomas de Depressão de Beck (Beck Depression Inventory).

${ }^{10}$ Obtido por meio da Escala de Sintomas de Ansiedade de Beck (Beck Anxiety Inventory). 


\section{RESULTADOS}

O perfil dos estudantes de Medicina membros e não membros do Gedaam pode ser observado na Tabela 1. Para os dois grupos, destaca-se a predominância de estudantes regulares no curso, de cor da pele branca, com orientação heterossexual, que não recebem bolsa de auxílio da universidade e que possuem pior percepção sobre a qualidade de vida autorreferida. Quando os grupos são comparados, diferenças estatisticamente significativas $(p<0,05)$ são observadas para as variáveis ciclo do curso, sexo, orientação sexual, recebimento de bolsas de auxilio e prática de atividade física regular.

Os resultados relacionados à percepção de suporte social demonstram que, em todos os domínios da ESSS, o grupo dos não membros do Gedaam apresentou escores médios ligeiramente mais altos que o dos que participaram da mentoria. Essa diferença foi estatisticamente significativa para os domínios satisfação com amigos, intimidade e satisfação com a família ( $p<0,05)$. No entanto, é possível observar que os escores médios para o domínio satisfação com atividades sociais não apresentaram diferenças estatisticamente significativas entre os dois grupos (Tabela 2).

$\mathrm{O}$ ambiente educacional foi avaliado pelos dois grupos como tendo "muitos problemas" (escore 95,51 para membros Gedaam e 100,05 para não membros), segundo o guia prático de interpretação da escala DREEM. Observou-se diferença estatisticamente significativa entre os dois grupos para a escala global e para todos os domínios, exceto Atmosfera. Em geral, todos os escores médios foram menores entre os membros do Gedaam em comparação com os não membros (Tabela 3).

Tabela 2. Médias e desvios padrão dos itens, dos domínios e da escala global da Escala de Satisfação com o Suporte Social (ESSS) entre estudantes de Medicina membros e não membros do Gedaam1 que responderam ao QualiMed, Universidade Federal de Minas Gerais, 2018

\section{Itens da Escala de Satisfação com o Suporte Social}

\section{Membros do Gedaam²}

\begin{tabular}{|c|c|c|c|c|c|}
\hline \multirow[t]{2}{*}{ Itens da Escala de Satisfação com o Suporte Social } & \multicolumn{2}{|c|}{$\operatorname{Sim}(n=347)$} & \multicolumn{3}{|c|}{ Não (n = 1.123) } \\
\hline & Média & DP & Média & DP & $p^{3}$ \\
\hline . Os amigos não me procuram tantas vezes quanto eu gostaria. & 3,11 & 1,23 & 3,32 & 1,27 & 0,007 \\
\hline 2. Estou satisfeito com a quantidade de amigos que tenho. & 3,97 & 1,19 & 4,01 & 1,19 & 0,668 \\
\hline 3. Estou satisfeito com a quantidade de tempo que passo com os meus amigos. & 2,95 & 1,31 & 3,00 & 1,28 & 0,521 \\
\hline 4. Estou satisfeito com as atividades e coisas que faço com o meu grupo de amigos. & 3,38 & 1,24 & 3,49 & 1,21 & 0,148 \\
\hline 5. Estou satisfeito com o tipo de amigos que tenho. & 4,14 & 1,05 & 4,27 & 1,00 & 0,036 \\
\hline 6. Por vezes sinto-me só no mundo e sem apoio. & 3,12 & 1,34 & 3,36 & 1,39 & 0,006 \\
\hline $\begin{array}{l}\text { 7. Quando preciso desabafar com alguém, encontro facilmente amigos com } \\
\text { quem o fazer. }\end{array}$ & 3,68 & 1,25 & 3,76 & 1,26 & 0,314 \\
\hline $\begin{array}{l}\text { 8. Mesmo nas situações mais embaraçosas, se precisar de apoio de } \\
\text { emergência, tenho várias pessoas a quem posso recorrer. }\end{array}$ & 3,78 & 1,19 & 3,87 & 1,19 & 0,181 \\
\hline $\begin{array}{l}\text { 9. Às vezes sinto falta de alguém verdadeiramente íntimo que me } \\
\text { compreenda e com quem possa desabafar sobre coisas íntimas. }\end{array}$ & 2,97 & 1,51 & 3,34 & 1,51 & $<0,001$ \\
\hline 10. Estou satisfeito com a forma como me relaciono com a minha família. & 3,54 & 1,23 & 3,81 & 1,19 & $<0,001$ \\
\hline 11. Estou satisfeito com a quantidade de tempo que passo com a minha família. & 2,54 & 1,29 & 2,69 & 1,32 & 0,053 \\
\hline 12. Estou satisfeito com o que faço em conjunto com a minha família. & 3,15 & 1,29 & 3,33 & 1,33 & 0,029 \\
\hline 13. Não saio com amigos tantas vezes quanto eu gostaria. & 2,41 & 1,34 & 2,42 & 1,32 & 0,912 \\
\hline 14. Sinto falta de atividades sociais que me satisfaçam. & 2,42 & 1,29 & 2,54 & 1,34 & 0,171 \\
\hline $\begin{array}{l}\text { 15. Gostava de participar mais em atividades de organizações (ex. clubes } \\
\text { desportivos, escoteiros, partidos políticos). }\end{array}$ & 2,95 & 1,45 & 3,06 & 1,45 & 0,200 \\
\hline
\end{tabular}

\section{Domínios ${ }^{4}$}

Satisfação com amigos

Satisfação com intimidade

Satisfação com família

Satisfação com atividades sociais

70,07

17,63

72,30

17,68

0,040

$67,56 \quad 21,04$

71,51

21,04

0,002

$61,50 \quad 20,24$

65,42

20,69

0,002

Escala global

$51,83 \quad 20,55$

53,39

21,23

0,229

63,97

66,93

15,23

0,002

\footnotetext{
${ }^{1}$ Gedaam: Grupo de Estudos em Didática Aplicada ao Aprendizado da Medicina.

${ }^{2}$ Estudantes que eram ou haviam sido membros do Gedaam entre os estudantes que responderam ao QualiMed.

${ }^{3}$ Valor-p obtido por meio do teste t de Student

${ }^{4}$ Transformados em escala de zero a 100. Quanto mais próximo de 100, melhor a percepção sobre suporte social.
} 
Tabela 3. Médias e desvios padrão dos domínios da escala Dundee Ready Education Environment Measure (DREEM) que avalia a percepção acerca do ambiente educacional entre estudantes de Medicina membros e não membros do Gedaam1 que responderam ao QualiMed, Universidade Federal de Minas Gerais, 2018

\begin{tabular}{ccccccc}
\hline & \multicolumn{5}{c}{ Membros do Gedaam } \\
\cline { 2 - 6 } & Domínios & \multicolumn{2}{c}{ Sim $(\mathbf{n}=\mathbf{3 4 7})$} & \multicolumn{3}{c}{ Não $(\mathbf{n}=\mathbf{1 . 1 2 3})$} \\
\cline { 2 - 6 } & Média & DP & Média & DP & $\mathbf{p}^{\mathbf{3}}$ \\
\hline Aprendizado & 20,41 & 6,59 & 21,28 & 6,94 & 0,039 \\
& Professores & 21,17 & 6,81 & 22,46 & 6,90 & 0,002 \\
& Acadêmico & 15,17 & 4,75 & 16,41 & 5,13 & $<0,001$ \\
& Atmosfera & 25,02 & 6,92 & 25,54 & 7,52 & 0,248 \\
& Social & 13,73 & 4,35 & 14,35 & 4,56 & 0,026 \\
\hline Escala global & & & & & \\
\multicolumn{2}{c}{ Satisfação com o ambiente de ensino } & 95,51 & 24,11 & 100,05 & 26,10 & 0,004 \\
\hline
\end{tabular}

1 Gedaam: Grupo de Estudos em Didática Aplicada ao Aprendizado da Medicina.

${ }^{2}$ Estudantes que eram ou haviam sido membros do Gedaam entre os estudantes que responderam ao QualiMed.

${ }^{3}$ Valor-p obtido por meio do teste t de Student.

\section{DISCUSSÃO}

Quando se analisa o perfil dos respondentes da pesquisa, pode-se perceber que o Gedaam apresentou maior porcentagem de estudantes de cor da pele não branca, de orientação não heterossexual e que recebem bolsa de auxílio da universidade em comparação com discentes não membros. Sabe-se que, apesar das conquistas com a adoção de políticas de acesso ao ensino superior pelo governo federal, esses grupos ainda sofrem segregação dentro da universidade ${ }^{18}$. Nesse contexto, o Gedaam pode funcionar como um espaço de apoio para grupos tradicionalmente marginalizados, principalmente em cursos elitistas como o de Medicina.

De forma geral, os estudantes dos dois grupos se mostraram mais satisfeitos que insatisfeitos com o suporte social, com escores acima de 60 para todos os domínios da ESSS, excetuando o domínio atividades sociais (escore $=51,83$ para membros e 53,39 para não membros do Gedaam). Esses resultados foram melhores que os encontrados em um estudo com estudantes de graduação portugueses, entre os quais os escores foram mais baixos, exceto para o domínio atividades sociais, em que os discentes tiveram escore igual a $70,82^{19}$.

Vale destacar que os participantes do programa de mentoria entre pares apresentaram escores menores que seus colegas que não participaram do Gedaam. Todos os domínios da ESSS, com exceção de atividades sociais, mostraram diferenças estatisticamente significativas entre participantes e não participantes. Esses resultados podem sinalizar que, de alguma maneira, estudantes com uma pior percepção de suporte social procuram a mentoria entre pares. Tendo esse aspecto como um objetivo central do grupo, o Gedaam se configura como um espaço inclusivo capaz de proporcionar aos estudantes terreno fértil para que novas relações e conexões sejam feitas. É importante ressaltar que o possível distanciamento do núcleo familiar e de amizades provocado pelo ingresso no ensino superior pode impactar a satisfação com o suporte social.

Nesse sentido, a mentoria entre pares exerce papel tanto no desenvolvimento acadêmico e profissional do mentorando como no âmbito pessoal. Destaca-se o compromisso do mentor em motivar o mentorando, norteá-lo em relação a fatores estressores no meio acadêmico e sobre o equilíbrio entre atividades pessoais e educacionais, além de assumir a posição de exemplo positivo ${ }^{20}$. O mentor não só ocupa uma posição de guia acadêmico, mas também de suporte social para o mentorando. Assim, pode-se aventar que os resultados encontrados no presente estudo são advindos da busca da mentoria, por parte dos estudantes com percepção ruim acerca de seu suporte social, a fim de melhorarem o nível dessa percepção a partir do sentimento de pertencimento construído pelo grupo do Gedaam.

Diferentemente dos outros três domínios da ESSS, não foram observadas diferenças estatisticamente significativas entre membros e não membros do Gedaam para o domínio satisfação com atividades sociais. Acredita-se que isso possa ser explicado pela extensa carga horária do curso de Medicina, que afeta negativamente a percepção das atividades sociais por ambos os grupos estudados.

De acordo com as Diretrizes Curriculares Nacionais de 2014, a carga horária mínima do curso de graduação em Medicina é de 7.200 horas com um limite mínimo de seis anos para integralização, permitindo grande variação na duração deste pelo país. Apresenta-se como o curso de maior carga horária do Brasil quando comparado a outros 
cursos de graduação, em que o número de horas-aula varia de 2.400 a quatro $\mathrm{mil}^{21}$.

Na UFMG, o curso de bacharelado em Medicina possui 8.085 horas de duração distribuídas nos seis anos. Em outras escolas médicas, como na Universidade de São Paulo (USP) e Universidade Federal de Santa Catarina (UFSC), são necessárias mais de nove mil horas-aula para esse mesmo curso. É preciso considerar que, além da carga horária obrigatória, o estudante despende tempo com o chamado currículo oculto, que inclui, além do estudo individual, a realização de diversas atividades extracurriculares, como monitorias, pesquisa e outros. Assim, o tempo para a prática de exercícios físicos, a manutenção do sono e as atividades de lazer e sociais fica prejudicado. Isso afeta igualmente os estudantes, independentemente da participação em grupos de mentoria, contribuindo também para uma pior avaliação do suporte social e da qualidade de vida, e para a alta prevalência de problemas de saúde mental em estudantes de Medicina ${ }^{3}$.

A avaliação do ambiente educacional pelos estudantes mostrou que, em geral, a avaliação do ambiente de ensino possui "muitos problemas", de acordo com o guia de análise do DREEM ${ }^{17}$. Essa avaliação foi particularmente mais incisiva no grupo dos que participaram da mentoria entre pares, com escore global menor se comparado aos não participantes. Isso foi observado tanto para a escala global quanto para os domínios. Esse achado pode sugerir que estudantes mais críticos em relação ao ambiente acadêmico buscam a mentoria entre pares como alternativa ao ensino tradicional praticado pela universidade. Além disso, por participarem do Gedaam, os membros se tornam mais exigentes em relação aos diversos aspectos que envolvem o ensino da Medicina. Por se tratar de um grupo que utiliza metodologias ativas de aprendizagem, como é o caso da discussão de casos clínicos pautada na medicina baseada em evidências que busca incentivar a participação ativa dos seus membros e os expõe a uma gama de possibilidades acadêmicas e pessoais, a participação nesse grupo pode gerar uma insatisfação maior com o ensino tradicional. Um estudo chileno com estudantes de Medicina mostrou que a modificação da percepção do ambiente educacional com pontuação mais positiva aconteceu após a mudança da metodologia tradicional para a ativa ${ }^{22}$.

Avaliações com o DREEM têm sido destacadas como preciosas para identificar áreas problemáticas e necessidades de treinamento do corpo docente, e apontar estratégias que reduzam as deficiências ${ }^{23}$.

Não foi objetivo deste trabalho determinar a causalidade entre a participação no Gedaam e os aspectos analisados. No entanto, a utilização do estudo transversal foi importante para conhecer o perfil de uma amostra dos estudantes que participam ou já participaram da mentoria entre pares, no que tange à sua percepção de suporte social e ambiente de ensino. A junção de dois bancos de dados mostrou-se um recurso interessante, acessível e oportuno.

\section{CONCLUSÃO}

Os resultados encontrados neste estudo fornecem informações valiosas quanto à percepção sobre os ambientes educacional e de suporte social de estudantes de Medicina de uma instituição pública. Embora não seja possível, a partir desses dados, compreender o que leva os alunos a procurar ou não a mentoria entre pares, pôde-se observar um perfil mais crítico em relação aos aspectos avaliados entre os discentes participantes.

Quando se analisa o perfil dos participantes do Gedaam, é possível perceber que a maioria estava nos anos iniciais do curso, momento de mais insegurança e incertezas quanto à nova forma de estudar e aprender e de maior fragilidade quanto ao distanciamento de familiares e amigos. Nesse sentido e entendendo a mentoria entre pares como uma ponte entre o discente e a universidade, essa modalidade de mentoria pode auxiliar de forma positiva o percurso dos estudantes, seja tornando-os mais críticos em relação ao ensino e à sua forma de aprender, seja fornecendo ferramentas de apoio acadêmico, como técnicas de estudo e gerenciamento de tempo, seja expondo-os à aquisição de novas habilidades sociais.

Sugerem-se, então, novas pesquisas, de cunho qualitativo, sobre esse tema que busquem entender não apenas a percepção dos alunos sobre o suporte social, mas também as motivações deles perante a mentoria e os grupos de apoio social.

\section{CONTRIBUIÇÃO DOS AUTORES}

Todos os autores participaram igualmente de todas as etapas de construção do artigo, incluindo a concepção, a análise de dados, a escrita e a revisão da versão final.

\section{CONFLITO DE INTERESSES}

Os autores declaram não haver conflito de interesses.

\section{FINANCIAMENTO}

Declaramos que não houve financiamento para a realização desta pesquisa.

\section{REFERÊNCIAS}

1. Rotenstein LS, Ramos MA, Torre M, Segal JB, Peluso MJ, Guille C, et al. Prevalence of depression, depressive symptoms, and suicidal ideation among medical students: a systematic review and meta-analysis. JAMA. 2016;316(21):2214-36. 
2. Yahaya SN, Wahab SFA, Yusoff MSB, Yasin MAM, Rahman MAA. Prevalence and associated factors of stress, anxiety and depression among emergency medical officers in Malaysian hospitals. World J Emerg Med. 2018;9(3):178-86.

3. Pacheco JP, Giacomin HT, Tam WW, Ribeiro TB, Arab C, Bezerra IM, et al. Mental health problems among medical students in Brazil: a systematic review and meta-analysis. Braz J Psychiatry. 2017;39(4):369-78.

4. Hill MR, Goicochea S, Merlo LJ. In their own words: stressors facing medical students in the millennial generation. Med Educ Online. 2018;23(1): 1530558.

5. Franzoi MAH, Martins G. Experiência de mentoring entre estudantes de graduação em enfermagem: reflexões e ressonâncias dialógicas. Interface Comun Saúde Educ. 2020;24:e190772.

6. Carragher J, McGaughey J. The effectiveness of peer mentoring in promoting a positive transition to higher education for first-year undergraduate students: a mixed methods systematic review protocol. Syst Rev. 2016;5(1):68.

7. Gilmour JA, Kopeikin A, Douché J. Student nurses as peer-mentors: collegiality in practice. Nurse Educ Pract. 2007;7(1):36-43.

8. Akinla O, Hagan P, Atiomo W. A systematic review of the literature describing the outcomes of near-peer mentoring programs for first year medical students. BMC Med Educ. 2018;18(1):98.

9. Souza MG, Reato LFN, Bellodi PL. Ressignificando a relação entre calouros e veteranos: mentoria de pares na visão de alunos mentores. Rev Bras Educ Med. 2020;44(4):e174.

10. Nimmons $D$, Giny $S$, Rosenthal J. Medical student mentoring programs: current insights. Adv Med Educ Pract. 2019;10:113-23.

11. Barker TA, Ngwenya N, Morley D, Jones E, Thomas CP, Coleman JJ. Hidden benefits of a peer-mentored "Hospital Orientation Day": first-year medical students' perspectives. Med Teach. 2012;34(4):e229-35.

12. Moreira SNT, Albuquerque ICS, Pinto Junior FEL, Gomes AHB. Programa de Mentoria do Curso de Medicina da Universidade Federal do Rio Grande do Norte: atividades integrativas em foco. Rev Bras Educ Med. 2020;44(4):e169.

13. Ornellas J. Suporte social: origens, conceitos e áreas de investigação. Anál Psicol. 1994:2-3(XII):333-9.
14. Ribeiro JLP. Escala de Satisfação com o Suporte Social (ESSS). Anál Psicol 1999;17(3):547-58.

15. Marôco JP, Campos JADB, Vinagre MG, Pais-Ribeiro JL. Adaptação transcultural Brasil-Portugal da Escala de Satisfação com o Suporte Social para Estudantes do Ensino Superior. Psicol Reflex Crit. 2014;27(2):247-56.

16. Oliveira Filho GR. Psychometric properties of Dundee Ready Educational Environment Measure (DREEM) applied to medical residentes. Med Teach. 2005;27(4):343-7.

17. Roff S, McAleer S, Harden RM, Al-Qahtani M, Ahmed AU, Deza H, et al Development and validation of the Dundee Ready Education Environment Measure (DREEM). Med Teach. 1997;(4):295-9.

18. Silva MLAM, Amaral E, Machado HC, Passeri SMRR, Bragança JF. Influência de políticas de ação afirmativa no perfil sociodemográfico de estudantes de Medicina de universidade brasileira. Rev Bras Educ Med. 2018;42(3):36-48

19. Anjos VNNF. Perceção do funcionamento familiar e do suporte social em estudantes do ensino superior em Portugal continental [dissertação]. Coimbra: Instituto Superior Miguel Torga; 2017.

20. American Psychological Association. Introduction to mentoring: a guide for mentors and mentees. Washington, DC: APA; 2006.

21. Brasil. Lei $n^{\circ} 4$, de 6 de abril de 2009. Dispõe sobre carga horária mínima e procedimentos relativos à integralização e duração dos cursos de graduação em Biomedicina, Ciência Biológicas, Educação Física, Enfermagem, Farmácia, Fisioterapia, Fonoaudiologia, Nutrição e Terapia Ocupacional, bacharelados, na modalidade presencial. Diário Oficial da União, Brasília, DF; abr 2009.

22. Riquelme A, Oporto M, Oporto J, Mendez Jl, Viviani P, Salech F, et al Measuring students' perception of the educational climate of the new curriculum at the Pontificia Universidad Católica de Chile: performance of the Spanish translation of the Dundee Ready Education Environment Measure (DREEM). Educ Health (Abingdon). 2009;22(1):112.

23. Bakhshialiabad H, Bakhshi G, Hashemi Z, Bakhshi A, Abazari F. Improving students' learning environment by DREEM: an educational experiment in an Iranian medical sciences university (2011-2016). BMC Med Educ. 2019;19(1):397. 